\title{
Téoros
}

Revue de recherche en tourisme

\section{La distribution par les prestataires de services}

\section{Le cas de l'hôtellerie}

\section{Marie-Claire Louillet}

Volume 9, numéro 3, novembre 1990

La distribution du produit touristique

URI : https://id.erudit.org/iderudit/1079884ar

DOI : https://doi.org/10.7202/1079884ar

Aller au sommaire du numéro

Éditeur(s)

Université du Québec à Montréal

ISSN

0712-8657 (imprimé)

1923-2705 (numérique)

Découvrir la revue

Citer cet article

Louillet, M.-C. (1990). La distribution par les prestataires de services : le cas de l’hôtellerie. Téoros, 9(3), 12-13. https://doi.org/10.7202/1079884ar d'utilisation que vous pouvez consulter en ligne.

https://apropos.erudit.org/fr/usagers/politique-dutilisation/ 


\section{La distribution par les prestataires de services}

Avec 1'apparition des chaînes hôtelières, l'industrie a vécu un changement structurel important notamment dans son mode de commercialisation. C'est à ce moment là que les centrales de réservations se sont développées et que les hôteliers ont réalisé 1 'importance de leur service de vente. Il ne suffisait plus d'accueillir chaleureusement le client de passage, encore fallait-il le démarcher. Aujourd'hui, quelles sont les méthodes utilisées par les hóteliers pour vendre efficacement leurs chambres? Dans un climat de plus en plus concurrentiel, que vont devenir les rapports existant entre les hôteliers et leurs partenaires commerciaux de longue date, les agents de voyages et les compagnies aériennes? Quel sera l'impact des progrès technologiques sur le mode de commercialisation?

\section{La vente directe}

L'hôtellerie, industrie de services, se différencie radicalement de toute industrie manufacturière car sa nature même englobe la production et la livraison simultanée de biens de consommation tels un repas, un séjour agréable et d'autres bénéfices intangibles. La quasi simultanéité existantentre la production et la livraison des différents services offerts impose un certain nombre


mode de commercialisation.

L'hôtelier ne vend pas des chambres mais une nuit de sommeil dans un cadre propice a la détente, dans un site enchanteur, dans un environnement de grande classe ou tout simplement dans un lieu central et fonctionnel. Pour ce faire, il doit convaincre son futur client que son établissement offre les avantages uniques qui correspondent aux besoins spécifíques de celui-ci, d'où la nécessité d'un recours quasi exclusif à la force de vente directe permettant la personnalisation et la concrétisation du produit.

Deplus, laparticipation de l'acheteur, directementet activement sollicitée par l' hôtelier lors de la vente, permet à ce dernier

\footnotetext{
- Madame Marie-Claire Louillet occupe le poste de Directriçe générale adjointe au Montréal Bonaventure Hilton,
}

d'intégrer rapidement les attentes de sa clientèle aux services offerts, favorisant ainsi l'évolution dynamique du produit. Dans le contexte économique actuel, le concept gagnant sera celui qui répond le mieux aux attentes de son segment de marché.

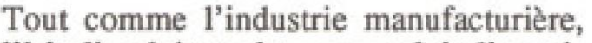
l'hôtelier doit rendre son produit disponible au moment et à l'endroit où le client est prêt à l'acheter. La force des chaînes hôtelières repose sur l'organisation de leur réseau de distribution composé principalement d'une centrale de réservations et d'une équipe de vente régionale. Les établissements indépendants, ne pouvant assumer seuls un tel coutt, se regroupent fréquemment en association afin d'assurer une meilleure commercialisation de leur produitpar le biais d'une présence accrue surle marché; tel estl'objectif premier des Relais et Châteawx, Des relais du silence, de la chaîne Hôte, et de la chaîne Romantik.

Le client, ne pouvant pas échantillonner ni visionner la qualité du produit, basera sa décision d'achat sur la réputation de l'établissement (ou de sa marque de commerce pour les chaînes hôtelières) ou bien encore, le plus souvent, se laissera influencer par la représentativité des vendeurs et des agents de réservations. En hôtellerie, la relation de confiance, sans laquelle la vente ne pourrait être conclue, doit être induite par le réseau de distribution et non par l'emballage, la texture ou la composition même du produit comme c'est le cas dans 1 'industrie manufacturière.

\section{L'appui d'une centrale de réservations}

Nous proposons d'examiner maintenant les éléments sur lesquels reposent le succès d"une telle transaction en prenant plus particulièrement l'exemple d'une centrale de réservations.

Le système utilisé doit, d'une part permettre de recueillir avec précision toutes les données qui traduisent les besoins des clients (dates de séjour, orthographe du nom, etc.) sans jamais perdre de vuela personnalisation de la communication. D'autre part, ce système doit aussi répondre avec courtoisie aux demandes de $\mathrm{l}^{\prime}$ acheteur en lui fournissant le plus d'informations possibles sur l'hôtel (c'est-à-dire types de chambres, de salles de réunion, tarifs, etc.) mais aussi toute autre information pertinente à son séjour (activités culturelles, sportives, moyens de transports, distances à parcourir, etc.). Finalement, la transaction sera conclue avec succès quand le client aura obtenu un numéro de confirmation, voire une copie écrite de sa réservation.

Comme nous pouvons le constater, un tel processus nécessitera un logiciel offrant une certaine flexibilité d'utilisation et permettant une mise à jour rapide de l'information contenue.

Il sera d'autant plus facile de livrer une excellente prestation de services si tous les besoins du client sont clairement identifiés au moment de la prise de commande. Par contre, l'objectif est surtout d'offrir un service rapide et personnalisé grâce à l'utilisation de l'historique client et de l'historique agent de voyages ou compagnie qui évitent la répétition du nom, de l'adresse, etc., pour les clients réguliers.

A l'heure actuelle, si laplupart des logiciels utilisés par les centrales de réservations permettent une communication précise et détaillée, I'intégration d'une banque de données centrale (historique client) en est encore au stade experimental ou ne concerne qu'un très petit nombre d'utilisateurs.

Un grand nombre de réseaux ne sont pas encore directement reliés au système informatique interne de l'hôtel et nécessitent une intervention manuelle afin de retranscrire les réservations; des erreurs peuvent se produire mais surtout l'hotelier n'obtient pas, en temps réel, une image exacte de la disponibilité des chambres.

D'après l'étude réalisée par W.J. Quain et P.W. Hermann ${ }^{(1)}$ auprès des centrales de réservations des chaînes aux États-Unis, l'utilisation des techniques de vente de base par les employés est très limitée et irrégulière. Cela peut s'expliquer par un manque de formation, une mauvaise connaissance du produit et la complexité des logiciels. De plus, l'hôtelier n'est pas 
capable d'évaluer l'importance réelle dece phénomène car, s'il reçoit la plupart du temps des rapports de production par bureau, il ignore souvent combien de demandes ont été reçues par ce mểme bureau et ne peut donc déterminer le taux de conversion, seule mesure de l'efficacité réelle. Leréseau Hilton Reservation Service, par exemple, fournit des statistiques des demandes de réservations non honorées, ventilées par raison: chambres non disponibles, tarif offert trop élevé, emplacement inadéquat, etc..

L'obtention et l'analyse de ce genre d'informations devient un outil de gestion indispensable dans le contexteéconomique actuel.

Une chambre d'hôtel, une table dans un restaurant sont des produits périssables qui ne peuvent pas être stockés pour faire face à la fluctuation de la demande. Par conséquent, au travers de son réseau de distribution, l'hotelier essaiera d'influencer la demande afin qu'elle corresponde mieux en tout temps à sa capacité. Il cherchera à modifier en temps réel le nombre de chambres à vendre par tarifs ou par segment de marché; cela implique que son hôtel soit relié de façon interactive au système de réservations. C'est le cas des grandes chaînes hôtelières; toutefois, certains logiciels offrent plus de souplesse que d'autres.

La possibilité d'opérer une liste d'attente, la recherche d'une solution alternative (c'est-à-dire autres dates, autres forfaits, hôtel le plus proche') sont autant d'atouts commerciaux qui seront nécessaires demain pour fidéliser une clientèle.

Toutefois, malgré l'évolution rapide de la technologie, l'hôtelier n'est pas le seul impliqué dans la distribution de son produit. Ses partenaires de longue date sont les agents de voyages et les réseaux de réservations des compagnies aériennes.

\section{Un nécessaire mariage de raison}

Les compagnies d'aviation, afin de vendre leur produit, ont êté les premières à créer leurs réseaux de communication (ARINC pour U.S. Airlines; etSITA/SAHARA pour l'Europe) età équiper les agents de voyages de terminaux et de logiciels de réservations; par exemple les systèmes de réservations suivants sont reliés à ARINC: Sabre (American Airlines), Apollo (United), Datas II (Delta), Panamac (Pan Am), et Pars (TWA). Afin de se rendre accessible aux agents de voyages, un hôtel doit être listé dans la plupart des logiciels de réservations des compagnies aériennes. Donc une cen- trale de réservations hôtelière consiste en un réseau reliant les hôtels entre eux et aux deux principaux réseaux existants: ARINC et SITA. Les compagnies aériennes, en vendant l'accès à leurs logiciels aux hôteliers, peuvent amortir les couts d'exploitation de leurs réseaux de communication.

Étant donné la diversité des logiciels utilisés par les agents de voyages, l' hôtelier doit être listé dans plusieurs systèmes, ce qui implique de multiples contrats commerciaux, entrées de données et mises à jour de l'information. Une grande partie des coûts reliếs à l'exploitation d'une centrale de réservations échappe donc totalement au contrôle des chaînes hôtelières: il en est ainsi des redevances dues à la compagnie aérienne et des commissions aux agences de voyages.

Alors que le rapport de forceentre les hôtels et les compagnies aériennes est celui d'un client vis-à-vis d'un fournisseur ayant le monopole, les agents de voyages sont en train de devenir des partenaires indispensables dans la conjoncture économique actuelle.

Leur force repose sur la nature intangible du service hôtelier et la relation de confiance existant entre le client et l'agent de voyages; ce dernier a une grande influence sur la sélection d'hôtel. En basse saison ou lors d'une ouverture, l'hôtelier est tenté d'offrir à son partenaire une commission plus élevée (15\% ou $20 \%$ du prix de la chambre au lieu du traditionnel $10 \%$ ), ou même d'en élargir l'assiette (commission payée sur la location de salons, les repas).

Les campagnes telles que "Votre commission est payée dans les 7 jours qui suivent le départ du client ou nous la doublons ${ }^{n(2)}$ se multiplient. Ces nouvelles initiatives permettent sans aucun doute de gagner rapidement une plus grande part de marché mais, une fois que la pratique segénéralise, les hôteliers verront seulement augmenter leur coutt de distribution.

D'autre part, les grandes compagnies continuent la rationalisation de leurs dépenses: négociation de tarifs corporatifs aveç un ou deux établissements seulement mais en jouant la carte du volume pour obtenir des prix plus bas, réduction des montants autorisés pour les repas et surtout la boisson, et enfin ré́organisation de leur département voyages. L'étape suivante consiste à confier aux agents de voyages toute la gestion des frais de déplacement; ce qui fait que l'hôtelier voit la proportion des nuitées commissionnables augmenter et le coût échapper de plus en plus à son contrôle.
En Europe, le groupe Accor acréé sa propre division tour-opérateur Accor Loisir, et sa centrale Res-Inter devrait être accessible sur Minitel en début d'année. De telles stratégies de développement permettrontelles à Accor de conserver un certain contrôle sur les coûts de commercialisation?

Comment les progrès de la télématique et l'accès possible à un nombre illimite de circuits vidéo vont-il influencer la distribution du produit hôtelier?

L'écran vidéo représente un média privilégié pour un hôtel car il permet de personnaliser et concrétiser les services proposés, de transmettre l'atmosphère unique de chaque établissement. Toutefois, le client fera-t-il confiance à la technologie nouvelle ou souhaitera-t-il toujours entrer en contact avec un agent de voyages, ou un vendeur pour effectuer sa réservation?

Les compagnies aériennes (Sabre) et les grossistes en voyages (Jaguar) proposent déjà aux hôteliers l'accès à l'écran.

Les conditions économiques sont telles que l'environnement de demain s'annonce de plus en plus compétitif et rend aussi la tâche de l'hôtelier fort complexe. Doit-il continuer à commercialiser son produit comme à l'heure actuelle avec l'aide de l'agent de voyages et de la compagnie aérienne ou doit-il au contraire se tourner vers la télé́matique et rendre son produit disponible sur l'écran Minitel, Alex ou meme sur le système informatique de son acheteur?

Comme dans le passế, l'ultime décideur sera le client et l' hôtelier doit anticiper son comportement afin de continuer ầ distribuer son produit avec succès.

Plus le service offert est complexe (par exemple pour les groupes), plus la qualité du contact sera déterminante et devra induire la confiance que ce soit par l'intermédiaire d'un vendeur, d'une centrale de réservations ou d'un écran.

\section{Notes explicatives et bibliographie}

(1) WLLIAM J. Quain and Peter W. HERMANN, Chain Reservations Systems: What Hapoens when Guest Calls?, Cornell Quarterly.

(2) Campagne utilise par Hilton International depuis 2 ans dejâ

LEVITT, Theodore, The Marketing Imagination, The Free Press, MacMillan New York, 1993

RAPPORT DU GROUPE DE TRAVAIL, Tourisme ot tólámatique. Organisation mondiale du tourisme. 1985 